\title{
Many-body effects for excitonic high-order wave mixing in monolayer transition metal dichalcogenides
}

\author{
H. K. Avetissian, ${ }^{1}$ G. F. Mkrtchian $\odot,{ }^{1, *}$ and K. Z. Hatsagortsyan $\odot^{2}$ \\ ${ }^{1}$ Centre of Strong Fields Physics, Yerevan State University, 0025, Yerevan, Armenia \\ ${ }^{2}$ Max-Planck-Institut für Kernphysik, Saupfercheckweg 1, 69117 Heidelberg, Germany
}

(Received 5 February 2020; revised manuscript received 24 March 2020; accepted 26 March 2020; published 24 April 2020)

\begin{abstract}
A microscopic quantum theory describing the nonlinear and nonperturbative optical response of monolayer transition metal dichalcogenides (MTMDs) to a two-color strong laser field is developed within the dynamical Hartree-Fock approximation. Taking into account the recently recognized importance of MTMD twodimensional nanostructures for light-wave electronics and optical communication, we investigate the influence of many-body effects in the nonlinear optical response. The high-frequency component of the field resonantly generates tightly bound excitons, and the low-frequency strong field component induces excitonic high-order wave mixing/harmonic generation (HWM/HHG). The considerable enhancement of $\mathrm{HWM} / \mathrm{HHG}$ spectra due to many-body excitonic effects is observed, which modifies the relative contribution of the interband and intraband channels, and essentially reshapes the radiation spectra. Our analysis confirms the efficiency of HWM and HHG in MTMDs, which may provide a promising platform for ultrafast modulation of light for optical communication.
\end{abstract}

DOI: 10.1103/PhysRevResearch.2.023072

\section{INTRODUCTION}

Recently, there has been a growing interest in extending atomic high-order harmonic generation (HHG) $[1,2]$ and attosecond time-resolved spectroscopy [3,4] into bulk crystals [5-14] and nanostructures [15-26]. The progress of recent decades in the field of nanotechnology enabled the synthesis of a new class of optical materials, namely two-dimensional (2D) nanostructures with very high carrier mobility and extraordinary properties [27]. Among such materials, 2D semiconductors such as atomically thin monolayer transition metal dichalcogenides (MTMDs) $[28,29]$ are of great interest. These 2D semiconductors with a nontrivial topology of energy bands have demonstrated remarkable optical properties and efficient light-matter interaction [30-33] that are absent in their bulk crystals. In particular, MTMDs possess distinctive electronic properties, such as direct optically assessable energy gaps in the visible spectral region [30] and nontrivial Berry curvatures of energy bands [34]. As a result of a weak dielectric screening due to a 2D structure [35], excitonic effects are significant in their optical properties as in the linear [36-41] as well as in the perturbative nonlinear regimes [42-50], and one expects a similarly large contribution of many-body effects at extreme optical nonlinearities [51], in particular in HHG and high-order wave mixing (HWM).

\footnotetext{
*Corresponding author: mkrtchian@ysu.am

Published by the American Physical Society under the terms of the Creative Commons Attribution 4.0 International license. Further distribution of this work must maintain attribution to the author(s) and the published article's title, journal citation, and DOI.
}

HHG in solids originates either from the intraband electronic current [52] or from the interband transitions [9-11]. The latter are similar to atomic HHG and they are well described within the semiclassical three-step model, including electron-hole creation, their acceleration, and recollision. In bulk solids, this is realized as with a single-color $[5,8]$ as well as two-color laser fields [6,7]. In the latter case with semiconductors, excitons were created with a near-infrared laser field, followed by high-order sideband generation (HSG) driven by $\mathrm{THz}$ fields [53-55].

The recent experimental verification of HHG in 2D MTMDs [56] opened up a new avenue toward the nonperturbative nonlinear optics with MTMDs. Nonperturbative HHG up to 18th order in a MTMD was demonstrated recently in Ref. [57]. The HSG has been observed recently also in MTMDs [58], where the polarization of sidebands is employed for valleytronics, i.e., for the control of the valley pseudospin at a few femtosecond timescale. The same is proposed in [59] to achieve with an ultrashort laser pulse.

While the theoretical analyses in Refs. [56,57] are dominated by a single-particle picture, significant enhancement of the efficiency per layer of harmonics compared to the bulk one, which is a consequence of the strong many-body Coulomb interaction, is observed in [56]. Many-body effects are commonly large in 2D materials, e.g., in graphene [25], however they are especially dominant in MTMDs due to the extremely large energy gap and large excitonic binding energies. The latter property makes MTDMs a promising platform for ultrafast (femtosecond) modulation of light for optical communication.

In this paper, the impact of the many-body interaction on the HWM process in MTMDs is investigated. We consider interaction of a strong two-color laser field with MTMDs 
when HWM proceeds via bound exciton states. The highfrequency (HF) wave is in the one-photon resonance with the excitonic transition, while the low-frequency (LF) wave drives the nonlinear response of the created exciton via multiphoton channels. We develop a nonperturbative microscopic quantum theory of HWM taking into account the electronelectron Coulomb interaction within the self-consistent dynamical Hartree-Fock approximation [60]. A closed set of integrodifferential equations for the single-particle density matrix is derived, and is solved numerically. We analyze the influence of many-body Coulomb effects on the dynamics of charge carriers, and we investigate their role in shaping the HWM/HHG spectra.

\section{MEAN-FIELD THEORY}

We consider the interaction of a strong two-color laser field $\mathbf{E}(t)$ with an MTMD. The waves propagate in a perpendicular direction to the monolayer plane $(X Y)$ of the MTMD: $\mathbf{E}(t)=f_{1}(t) E_{01}\left(\hat{\mathbf{x}} \cos \omega_{1} t+\hat{\mathbf{y}} \zeta_{1} \sin \omega_{1} t\right)+$ $f_{2}(t) E_{02}\left(\hat{\mathbf{x}} \cos \omega_{2} t+\hat{\mathbf{y}} \zeta_{2} \sin \omega_{2} t\right)$, with the frequencies $\omega_{1,2}$, ellipticities $\zeta_{1,2}$, pulse durations $\tau_{1,2}$, and envelopes $f_{1,2}(t)=$ $\sin ^{2}\left(\pi t / \tau_{1,2}\right)$ of the two waves, respectively.

The dynamics of charge carriers is described by a single-particle density matrix. Let us introduce the applied mean-field dynamical Hartree-Fock approximation. We use the second quantization formalism, expanding the fermionic field operators on the basis eigenstates for the valence $(v)$ and conduction $(c)$ bands of a single-particle Hamiltonian: $\hat{\Psi}(\mathbf{r})=\sum_{\lambda, \mathbf{k}} \hat{e}_{\lambda \mathbf{k}}|\lambda ; \mathbf{k}\rangle e^{i \mathbf{k} \cdot \mathbf{r}}$, where $\hat{e}_{\lambda \mathbf{k}}\left(\hat{e}_{\lambda \mathbf{k}}^{\dagger}\right)$ are the annihilation (creation) operators for an electron with momentum $\mathbf{k}$, and $\lambda=\{b, s, \tau\}$ is a set of quantum numbers (band, spin, and valley). The single-particle Hamiltonian is based on a 2D, two-band, and $\mathbf{k} \cdot \mathbf{p}$ model up to second order in $k$, relative to $\pm K$ points, coupled to the strong laser field. (Details are provided in Appendix A.) The total Hamiltonian in the second quantization reads

$$
\hat{H}=\hat{H}_{\text {free }}+\hat{H}_{\mathrm{C}}+\hat{H}_{\text {int }},
$$

where

$$
\hat{H}_{\text {free }}=\sum_{\lambda, \mathbf{k}} \mathcal{E}_{\lambda}(\mathbf{k}) \hat{e}_{\lambda \mathbf{k}}^{\dagger} \hat{e}_{\lambda \mathbf{k}}
$$

is the free-particle Hamiltonian, with the single-particle eigenenergies $\mathcal{E}_{\lambda}(\mathbf{k})$,

$$
\hat{H}_{\text {int }}=-\mathbf{E}(t) \cdot \hat{\mathbf{P}}
$$

is the light-matter interaction Hamiltonian, with the polarization operator $\hat{\mathbf{P}}=-e \int d \mathbf{r} \hat{\Psi}^{\dagger}(\mathbf{r}) \mathbf{r} \hat{\Psi}(\mathbf{r})$, and $e$ is the elementary charge. The electron-electron Coulomb interaction is described by

$$
\hat{H}_{\mathrm{C}}=\frac{1}{2} \int d \mathbf{r} \int d \mathbf{r}^{\prime} \hat{\Psi}^{\dagger}(\mathbf{r}) \hat{\Psi}^{\dagger}\left(\mathbf{r}^{\prime}\right) V_{C}\left(\mathbf{r}-\mathbf{r}^{\prime}\right) \hat{\Psi}\left(\mathbf{r}^{\prime}\right) \hat{\Psi}(\mathbf{r}),
$$

with the effective potential $V_{C}(\mathbf{r})$. In the Hartree-Fock approximation, we reduce the Coulomb interaction Hamiltonian into the mean-field Hamiltonian:

$$
\hat{H}_{\mathrm{C}} \approx-\sum_{\lambda_{1} \lambda_{2}} \sum_{\mathbf{k}^{\prime}, \mathbf{k}} V_{2 \mathrm{D}}\left(\mathbf{k}-\mathbf{k}^{\prime}\right) \digamma_{\lambda_{1} \lambda_{2}}\left(\mathbf{k}^{\prime}, \mathbf{k}\right)\left\langle\left(\hat{e}_{\lambda_{2} ; \mathbf{k}^{\dagger}}^{\dagger} \hat{e}_{\lambda_{1} ; \mathbf{k}^{\prime}}\right) \hat{e}_{\lambda_{1} ; \mathbf{k}}^{\dagger} \hat{\mathbf{e}}_{\lambda_{2} ; \mathbf{k}},\right.
$$

where $V_{2 \mathrm{D}}(\mathbf{q})$ is the $2 \mathrm{D}$ interaction potential in momentum space, and $\digamma_{\lambda_{1} \lambda_{2}}\left(\mathbf{k}^{\prime}, \mathbf{k}\right)=\left\langle\lambda_{1} ; \mathbf{k}\right|$ $\left|\lambda_{1} ; \mathbf{k}^{\prime}\right\rangle\left\langle\lambda_{2} ; \mathbf{k}^{\prime} \| \lambda_{2} ; \mathbf{k}\right\rangle \delta_{s_{1} s_{2}} \delta_{\tau_{1} \tau_{2}}$ is the factor modifying the interaction potential. We obtain a closed set of equations for the dynamic mean-field parameters: the interband polarization $\mathcal{P}_{\eta}(\mathbf{k}, t)=\left\langle\hat{e}_{c, \eta ; \mathbf{k}}^{+}(t) \hat{e}_{v, \eta ; \mathbf{k}}(t)\right\rangle$, and the distribution functions for the conduction $\mathcal{N}_{c, \eta}(\mathbf{k}, t)=\left\langle\hat{e}_{c, \eta ; \mathbf{k}}^{+}(t) \hat{e}_{c, \eta ; \mathbf{k}}(t)\right\rangle$ and valence $\mathcal{N}_{v, \eta}(\mathbf{k}, t)=\left\langle\hat{e}_{v, \eta ; \mathbf{k}}^{+}(t) \hat{e}_{v, \eta ; \mathbf{k}}(t)\right\rangle$ bands, where $\eta=\{s, \tau\}$. On the Hartree-Fock level for an undoped system in equilibrium, the initial conditions $\mathcal{P}_{\eta}(\mathbf{k}, 0)=0, \quad N_{c, \eta}(\mathbf{k}, 0)=0$, and $N_{v, \eta}(\mathbf{k}, 0)=1$ are assumed, neglecting thermal occupations. Since $N_{v, \eta}(\mathbf{k}, t)=1-N_{c, \eta}(\mathbf{k}, t)$, the equation for $N_{v, \eta}(\mathbf{k}, t)$ is superficial. Thus, the MTMD interaction with a strong laser field is modeled as

$$
\begin{aligned}
i \hbar\left[\partial_{t}\right. & \left.-e \mathbf{E}(t) / \hbar \partial_{\mathbf{k}}\right] \mathcal{P}_{\eta}(\mathbf{k}, t)+\left[\tilde{\Delta}_{\eta}+\hbar^{2} \mathbf{k}^{2} / 2 \mu_{\eta}+i \hbar \Gamma_{\eta}\right. \\
& \left.+e \mathbf{E}(t) \delta \mathbf{D}_{\mathrm{B}}(\eta, \mathbf{k})-\boldsymbol{\Xi}_{c}\left(\mathbf{k}, t ; N_{c, \eta}\right)\right] \mathcal{P}_{\eta}(\mathbf{k}, t) \\
= & {\left[\mathbf{E}(t) \mathbf{D}_{\mathrm{tr}}(\eta, \mathbf{k})+\Omega_{c}\left(\mathbf{k}, t ; \mathcal{P}_{\eta}\right)\right]\left(1-2 N_{c, \eta}(\mathbf{k}, t)\right), }
\end{aligned}
$$

$$
\begin{aligned}
& i \hbar\left[\partial_{t}-e \mathbf{E}(t) / \hbar \partial_{\mathbf{k}}+\Gamma_{0 \eta}\right] N_{c, \eta}(\mathbf{k}, t) \\
& \quad=-\left[\mathbf{E}(t) \mathbf{D}_{\mathrm{tr}}^{*}(\eta, \mathbf{k})+\Omega_{c}^{*}\left(\mathbf{k}, t ; \mathcal{P}_{\eta}\right)\right] \mathcal{P}_{\eta}(\mathbf{k}, t)-\text { c.c. }
\end{aligned}
$$

where $\mu_{\eta}$ is the reduced mass of an electron-hole pair, $\tilde{\Delta}_{\eta}$ is the renormalized gap, and $\Gamma_{\eta}$ and $\Gamma_{0 \eta}$ are the phenomenological relaxation rates, which account for correlation terms neglected on the Hartree-Fock level; $\delta \mathbf{D}_{\mathrm{B}}(\eta ; \mathbf{k})=\left\langle c, \eta ; \mathbf{k}\left|i \partial_{\mathbf{k}}\right| c, \eta ; \mathbf{k}\right\rangle-\left\langle v, \eta ; \mathbf{k}\left|i \partial_{\mathbf{k}}\right| v, \eta ; \mathbf{k}\right\rangle \quad$ is the difference of the Berry connection of bands, and $\mathbf{D}_{\text {tr }}(\eta ; \mathbf{k})=-e\left\langle v, \eta ; \mathbf{k}\left|i \partial_{\mathbf{k}}\right| c, \eta ; \mathbf{k}\right\rangle$ is the interband transition dipole moment. The many-body Coulomb interaction renormalizes the light-matter coupling via the internal dipole field of all generated electron-hole excitations:

$$
\Omega_{c}\left(\mathbf{k}, t ; \mathcal{P}_{\eta}\right)=\sum_{\mathbf{k}^{\prime}} V_{2 \mathrm{D}}\left(\mathbf{k}-\mathbf{k}^{\prime}\right) \digamma_{v c ; \eta}\left(\mathbf{k}^{\prime}, \mathbf{k}\right) \mathcal{P}_{\eta}\left(\mathbf{k}^{\prime}, t\right),
$$

as well as the transition energies:

$$
\begin{aligned}
& \Xi_{c}\left(\mathbf{k}, t ; N_{c, \eta}\right) \\
& \quad=\sum_{\mathbf{k}^{\prime}} V_{2 \mathrm{D}}\left(\mathbf{k}-\mathbf{k}^{\prime}\right)\left[\digamma_{c c ; \eta}\left(\mathbf{k}^{\prime}, \mathbf{k}\right)+\digamma_{v v ; \eta}\left(\mathbf{k}^{\prime}, \mathbf{k}\right)\right] N_{c, \eta}\left(\mathbf{k}^{\prime}, t\right) .
\end{aligned}
$$

While our ansatz is similar to that of Refs. [7,58], our set of Eqs. (3) and (4) contains extra terms: dynamic energy modulation due to the Berry connection of bands, and the total electric field in the term $\sim \partial_{\mathbf{k}}$, rather than the external field only. The first one provides gauge invariance with respect to the phase transformation of Bloch functions $|\lambda ; \mathbf{k}\rangle$, important in the case of nontrivial Berry curvatures [34]. The second one has a considerable impact on the harmonics of the HF wave.

The interaction potential is modeled by the Keldysh potential $V_{2 \mathrm{D}}(\mathbf{k})=2 \pi e^{2} /\left[\epsilon|\mathbf{k}|\left(1+\rho_{0}|\mathbf{k}|\right)\right][61,62]$, which accounts for the substrate-induced screening in the $2 \mathrm{D}$ nanostructure with a thickness $d$. Here, $\epsilon \equiv\left(\epsilon_{1}+\epsilon_{2}\right) / 2$, with the dielectric constants of the above $\epsilon_{1}$ and below $\epsilon_{2}$ surrounding media, and $\rho_{0} \simeq 2 d \epsilon_{b} / \epsilon$ is the screening length, with the inplane component of the dielectric tensor of the bulk material $\epsilon_{b}[35]$. 
We will consider two excitation regimes: the lowintensity regime when $\left|\mathbf{E}(t) \mathbf{D}_{\text {tr }}(\eta, \mathbf{k})\right| \tau_{1,2} / \hbar \ll 1$, and the high-intensity one at $\left|\mathbf{E}(t) \mathbf{D}_{\text {tr }}(\eta, \mathbf{k})\right| \tau_{1,2} / \hbar \gtrsim 1$. The lowintensity regime corresponds to the Keldysh approximation [63], when $N_{c, \eta} \ll 1$. In the high-intensity regime, the density of excitons can reach large values, which induces dynamic screening of the Coulomb interaction in addition to the dielectric screening from the substrate. In the latter case, $V_{2 \mathrm{D}}(\mathbf{q})$ is replaced by $V_{2 \mathrm{D}}(\mathbf{q}) / \epsilon_{\mathbf{q}}(t)$, using the quasistatic Lindhard screening model $[64,65]$

$$
\begin{aligned}
\epsilon_{\mathbf{q}}(t)= & 1-V_{2 \mathrm{D}}(\mathbf{q}) \sum_{\lambda, \mathbf{k}} \digamma_{\lambda \lambda}(\mathbf{k}-\mathbf{q}, \mathbf{k}) \\
& \times \frac{\mathcal{N}_{\lambda}(\mathbf{k}-\mathbf{q}, t)-\mathcal{N}_{\lambda}(\mathbf{k}, t)}{\mathcal{E}_{\lambda}(\mathbf{k}-\mathbf{q})-\mathcal{E}_{\lambda}(\mathbf{k})} .
\end{aligned}
$$

Finally, HWM is determined by the interband

$$
\mathbf{j}_{\mathrm{e}}(t)=\sum_{\eta, \mathbf{k}}\left(\mathbf{D}_{\mathrm{tr}}^{*}(\eta ; \mathbf{k}) \frac{d \mathcal{P}_{\eta}(\mathbf{k}, t)}{d t}+e \widetilde{\mathbf{v}}_{\eta}^{*} \mathcal{P}_{\eta}(\mathbf{k}, t)\right)+\text { c.c. }
$$

and the intraband

$$
\mathbf{j}_{\mathrm{a}}(t)=-e \sum_{\eta, \mathbf{k}}\left(\delta \mathbf{D}_{\mathrm{B}}(\eta ; \mathbf{k}) \frac{d N_{c, \eta}}{d t}+\mathbf{v}_{\eta} N_{c, \eta}\right)
$$

currents, where $\quad \mathbf{v}_{\eta}=\hbar \mathbf{k} / \mu_{\eta}+\hbar^{-1} \partial_{\mathbf{k}}\left[e \mathbf{E}(t) \delta \mathbf{D}_{\mathrm{B}}(\eta, \mathbf{k})-\right.$ $\left.\Xi_{c}\left(\mathbf{k}, t ; N_{c, \eta}\right)\right]$ and $\tilde{\mathbf{v}}_{\eta}^{*}=\hbar^{-1} \partial_{\mathbf{k}}\left[\mathbf{E} \mathbf{D}_{\mathrm{tr}}^{*}(\eta ; \mathbf{k})+\Omega_{c}^{*}\left(\mathbf{k}, t ; \mathcal{P}_{\eta}\right)\right]$. For a sufficiently large 2D sample, the generated electrical field is proportional to the surface current: $\mathbf{E}^{(g)}(t)=-4 \pi\left[\mathbf{j}_{\mathbf{e}}(t)+\mathbf{j}_{\mathbf{a}}(t)\right] / c$. The HWM spectrum is obtained from the Fourier transform of the generated field.

\section{RESULTS}

We explore the nonlinear response of MTMDs in a twocolor laser field. As an exemplary MTMD material, $\mathrm{MoS}_{2}$ is considered on the $\mathrm{SiO}_{2}$ substrate $\left(\epsilon_{1}=1, \epsilon_{2}=3.9\right)$, or embedded in $\mathrm{hBN}\left(\epsilon_{1}=\epsilon_{2}=5\right)$ or the free-standing monolayer $\operatorname{MoS}_{2}\left(\epsilon_{1}=\epsilon_{2}=1\right)$. The Coulomb interaction increases from the first case to the third one. The renormalized gap for the $A$ excitonic branch $(s \tau=1)$ is $\tilde{\Delta}_{s \tau=1}=\Delta_{\text {opt }}-\mathcal{E}_{A}$, with the overall optical gap $\Delta_{\text {opt }}$ and the binding energy $\mathcal{E}_{A}$ of the $A$ exciton. To compare the results for the different substrates, we will keep fixed $\Delta_{\text {opt }}=1.9 \mathrm{eV}$ and change $\mathcal{E}_{A}$. The latter for the free-standing monolayer $\mathrm{MoS}_{2}$ is calculated to be $\mathcal{E}_{A} \simeq-0.5 \mathrm{eV}$ for the $\mathrm{SiO}_{2}$ substrate $\mathcal{E}_{A} \simeq-0.31 \mathrm{eV}$, and for $\mathrm{hBN} \mathcal{E}_{A} \simeq-0.16 \mathrm{eV}$. The excitons in MTMDs are built up from electron-hole pairs located at the $K\left(K^{\prime}\right)$ point in the first Brillouin zone, and they can be selectively addressed by left (right) -handed circularly polarized light. For concreteness, we consider the HF wave to be circularly polarized $\left(\zeta_{1}=1\right)$ and resonant to the excitonic transition $\left(\hbar \omega_{1}=\Delta_{\text {opt }}\right)$. The LF midinfrared wave $\left(\hbar \omega_{2}=0.1 \mathrm{eV}\right)$ is linearly polarized $\left(\zeta_{2}=0\right) ; \tau_{1}=\tau_{2}=40 \pi / \omega_{2}$.

Relevant local atomic units for energy, length, and electricfield strength are used, defined for an $A$ exciton on the $\mathrm{SiO}_{2}$ substrate as $\varepsilon_{\mathrm{a}}=\mu_{A} e^{4} / \hbar^{2} \epsilon^{2} \simeq 1.08 \mathrm{eV}, l_{\mathrm{a}}=\hbar^{2} \epsilon / \mu_{A} e^{2} \simeq$ $5.4 \times 10^{-8} \mathrm{~cm}$, and $E_{\mathrm{a}}=\varepsilon_{\mathrm{a}} / e l_{\mathrm{a}} \simeq 2 \times 10^{7} \mathrm{~V} / \mathrm{cm}$, respectively. The dephasing constants are chosen according to the experimental data: in the low-intensity regime, $\Gamma_{\eta}=50 \mathrm{meV}$

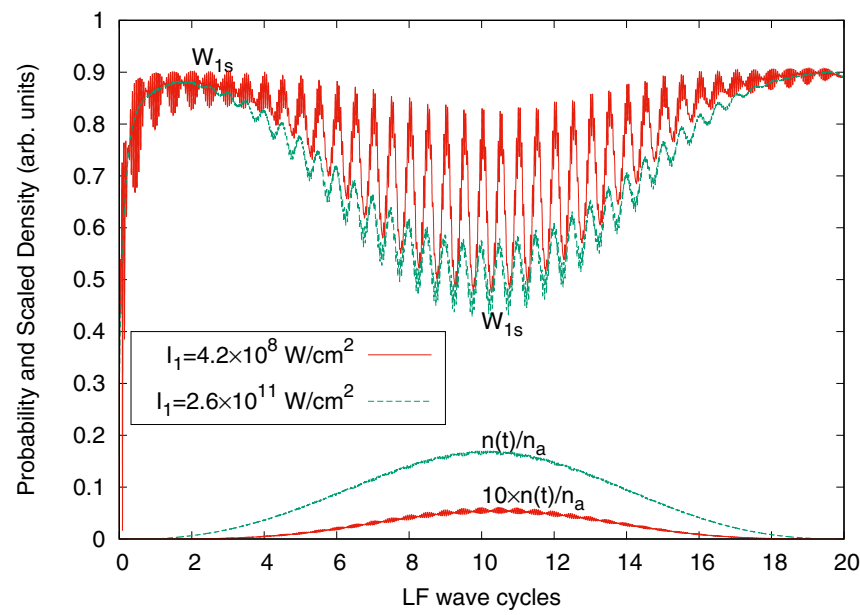

FIG. 1. Resonant creation of excitons in a monolayer $\mathrm{MoS}_{2}$ on $\mathrm{SiO}_{2}$ substrate. The probability to find the electron-hole pair in the $1 s$ excitonic state for the low-intensity regime (red-solid line) when $E_{01}=0.02$ 1.a.u. (laser intensity $I_{1}=4.2 \times 10^{8} \mathrm{~W} / \mathrm{cm}^{2}$ ) and for the high-intensity excitation regime (green-dashed line) when $E_{01}=0.5$ 1.a.u. $\left(I_{1}=2.6 \times 10^{11} \mathrm{~W} / \mathrm{cm}^{2}\right)$. In both cases, $E_{02}=0.046$ 1.a.u. $\left(I_{2}=2.2 \times 10^{9} \mathrm{~W} / \mathrm{cm}^{2}\right)$. The bottom lines show the density $n(t)$ of excitons (normalized to $n_{a}=1 / l_{\mathrm{a}}^{2}$ ) for both regimes.

and $\Gamma_{0 \eta}=0.1 \Gamma_{\eta}[30]$; in the high-intensity regime, $\Gamma_{0 \eta}=$ $\Gamma_{\eta}=75 \mathrm{meV}[65]$.

The resonant exciton creation and its further dynamics is shown in Fig. 1 via numerical solution of Eqs. (3) and (4) for $\mathrm{MoS}_{2}$ on the $\mathrm{SiO}_{2}$ substrate. The time evolution of the relative population of the excitonic $1 s$ state $W_{1 s}(t)=$ $\left|\left\langle\left|\varphi_{1 s}(\mathbf{k})\right|\left|\mathcal{P}_{1}(\mathbf{k}, t)\right|\right\rangle\right|^{2} /\left\langle\mathcal{P}_{1}(\mathbf{k}, t) \| \mathcal{P}_{1}(\mathbf{k}, t)\right\rangle$ and of the density of excitons $n(t)=\sum_{\lambda, \mathbf{k}} \mathcal{N}_{\lambda}(\mathbf{k}, t)$ is presented. As is seen, excitons are mainly created in the bound $1 s$-state. During interaction the $1 s$-state population oscillates, which corresponds to the ionization-recollision-recombination dynamics with a considerable depletion of the $1 s$-state in the high-intensity regime.

The density of excitons is a slowly varying function of time, which justifies the quasistatic approach to dynamic screening. In the electron-hole plasma, the screening is built up on a timescale given by the plasma oscillation period. The dynamic screening will set in when the plasma oscillation period is much smaller than the interaction time (pulse durations). For the 2D case, the plasma frequency is $\omega_{p}=$ $\sqrt{2 \pi e^{2} n q / \epsilon m}$ [66], where $m$ is the charged carrier effective mass $\left(m \simeq 2 \mu_{A}\right)$. From Fig. 1 one can see that the condition $\tau_{1,2} \gg 2 \pi / \omega_{p}$ is practically satisfied for the densities used in the high-intensity regime. Meanwhile, in the lowintensity regime, this condition can be violated. However, in the quasiequilibrium state, the screening wave number is proportional to the carrier occupation at the origin $\mathcal{N}_{c, \eta}(\mathbf{k}=\mathbf{0})$ [66], which is negligibly small in the low-intensity regime $\left(\mathcal{N}_{c, \eta} \ll 1\right)$. Thus, one can safely ignore dynamic screening in the low-intensity regime.

The radiation spectra for $\mathrm{MoS}_{2}$ for different substrates in a weak field limit are shown in Fig. 2(a). Due to the combined action of two waves, output radiation emerges at frequencies $\ell \omega_{1} \pm n \omega_{2}$. Since inversion symmetry is broken for MTMDs, 

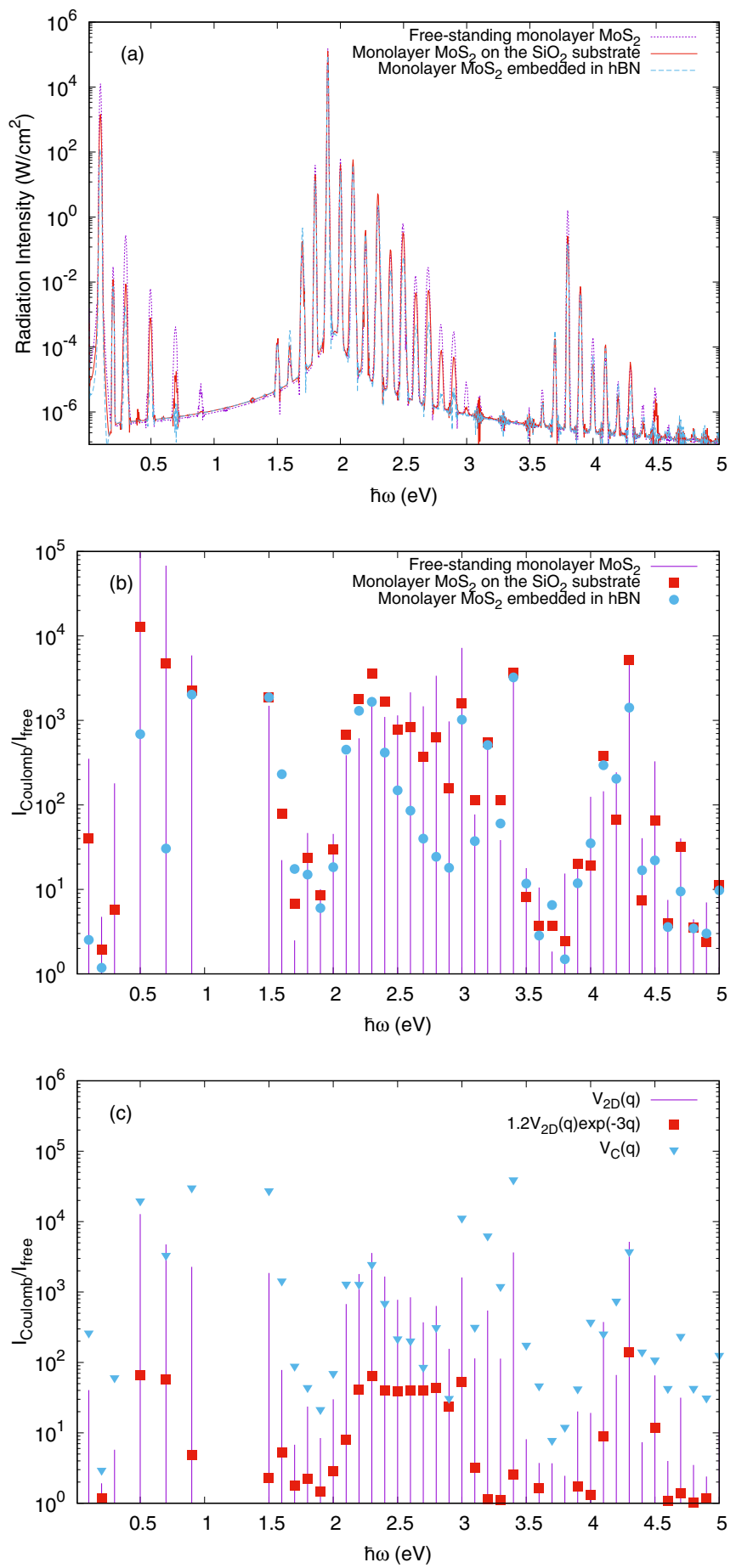

FIG. 2. HWM in the low-intensity regime: (a) radiation intensity via $\log _{10}\left[\left|\mathbf{E}^{(g)}(\omega)\right|^{2} /(4 \pi)\right]$ for the monolayer $\mathrm{MoS}_{2}$ on various substrates (see the legends) with different Coulomb interaction strength; (b) Coulomb enhancement via the ratio of the radiation intensities with and without Coulomb interaction (free quasiparticles) in the logarithmic scale; (c) Coulomb enhancement for $\mathrm{MoS}_{2}$ on $\mathrm{SiO}_{2}$ substrate with different potentials; $I_{1}=4.2 \times 10^{8} \mathrm{~W} / \mathrm{cm}^{2}$ and $I_{2}=$ $2.2 \times 10^{9} \mathrm{~W} / \mathrm{cm}^{2}$.

there is no restriction on the total number of involved photons and $\ell, n=0,1,2, \ldots$. The frequencies corresponding to $\ell=$ 0 and $n>0$ are dominantly generated by the intraband current, and they correspond to the electron-hole recombination into the final bound state, while $\ell>0$ corresponds to the electron-hole annihilation where the interband current is dominated. The spectrum shows harmonics of the HF wave $\left(\omega_{1}\right.$ and $\left.2 \omega_{1}\right)$, with high-order even- and odd-order sidebands of comparable strength, which is due to the trigonal warping effect (Appendix A). All spectral lines are enhanced with the increase of the Coulomb interaction. The enhancement factor can reach up to $10^{3}$, and it is not uniform across the spectrum; see Fig. 2(b). Thus, the HWM spectral shape is essentially modified due to many-body effects.

In contrast to the semiclassical three-step model of $\mathrm{HHG}$ [67], here we have four steps, which include resonant exciton creation, its ionization, electron-hole excursion in the continuum, and recollision. At the recollision, two outcomes are possible: recombination into the excitonic state $(\ell=0)$ or electron-hole annihilation $(\ell>0)$. For an MTMD, the Coulomb interaction has a significant contribution to all steps. In the first step, discrete excitonic states are created due to the electron-hole Coulomb interaction, which increases the density of states near the band edge, and consequently increases the probability of the excitation. In the second step, the Coulomb interaction is significant because the ionization proceeds in the nonadiabatic regime (the Keldysh parameter $\gamma_{K}=\omega_{2} \sqrt{\left|\mathcal{E}_{A}\right|} / E_{02} \sim 1$ [63]), and the electron appears in the continuum close to the hole. For the third electron-hole excursion step, the Coulomb interaction is also important, because the excursion length $\sim E_{02} / \omega_{2}^{2} \simeq 5 l_{\mathrm{a}}$ is of the order of the exciton radius. This is in contrast with HHG in bulk semiconductors [53-55], where the common Coulomb-free three-step model is a good approximation. The role of the Coulomb field for the fourth, recollision step of the accelerated electron-hole pair at large quasimomenta $q$ is analyzed using an artificial soft-core potential $1.2 e^{-3 q} V_{2 \mathrm{D}}(q)$ and pure Coulomb potential $V_{c}(q)=2 \pi \alpha e^{2} /[\epsilon|q|]$, with $\alpha=0.42$. In the soft-core potential, the Coulomb effect is switched off at large quasimomenta $q$, while for the pure Coulomb potential it is enhanced. However, the parameters of both potentials are tuned to reproduce the binding energy and radius of the exciton. Therefore, the Coulomb effects in the first three steps of the process are not disturbed in both potentials. The results of HWM for these potentials are shown in Fig. 2(c). As is seen, for the soft-core potential (pure Coulomb potential) the Coulomb enhancement effect is suppressed (increased) by roughly 10 times compared with the case of $V_{2 \mathrm{D}}(q)$, indicating the significant role of many-body effects for the recollision step. From Fig. 2(b) we see that the enhancement for $\ell=1$ (interband transitions) is larger by an order of magnitude than for $\ell=0$ (intraband transitions), because the latter is determined by the conduction-band filling, which in the weak-field regime $\mathcal{N}_{c, \eta}(\mathbf{k}, t) \ll 1$. Thus, the annihilation becomes more probable due to many-body effects than the recombination to the final bound excitonic state at the recollision.

Next, we consider a high-intensity regime for a strong HF wave when the population change is significant; see the spectra in Fig. 3(a) for MTMDs on $\mathrm{SiO}_{2}$. The HHG/HWM yield, and consequently the efficient harmonic number in this regime, are higher than in the low-intensity regime. In contrast to the low-intensity regime, here the Coulomb enhancement factor is generally larger for $\ell=0$ than for $\ell=1$, because of a large intraband current at the large population of the 

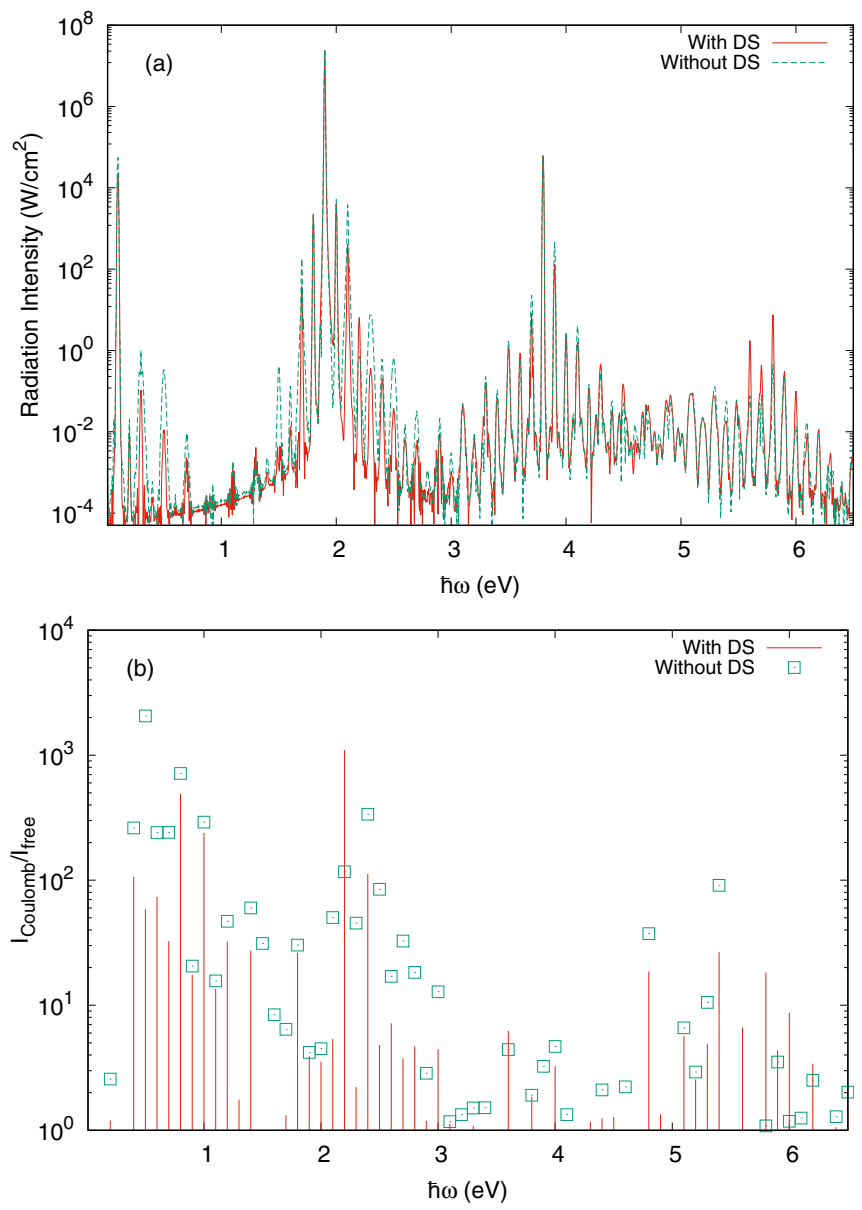

FIG. 3. HWM in the high-intensity regime: (a) radiation intensities for the monolayer $\mathrm{MoS}_{2}$ on $\mathrm{SiO}_{2}$ substrate with and without dynamic screening of the Coulomb interaction; (b) Coulomb enhancement via the ratio of the radiation intensities for $\mathrm{MoS}_{2}$ on $\mathrm{SiO}_{2}$ substrate with and without Coulomb interaction. The impulses show the ratio for the dynamically screened Coulomb potential. $I_{1}=2.6 \times 10^{11} \mathrm{~W} / \mathrm{cm}^{2}$ and $I_{2}=2.2 \times 10^{9} \mathrm{~W} / \mathrm{cm}^{2}$.

resonant valley $N_{c, \pm 1,1}(\mathbf{k}, t)_{\max } \sim 1$. Although there is significant Coulomb enhancement [see Fig. 3(b)], the dynamic screening reduces considerably the HWM yield, especially for recollisions with small momenta and for odd harmonics. In addition, in this case there are several additional competitive factors depending on the valley that alter HWM. The first is the band filling factor $1-2 N_{c, \eta}(\mathbf{k}, t)$ in Eq. (3), which is large for the resonant valley $(\tau=1)$. The phase space, which is occupied by surrounding electrons, is not available for the scattering due to Pauli blocking, which reduces the Coulomb interaction and the overall light-matter coupling strength. The next factor is the shrinkage of the band gap due to large $\Xi_{c}\left(\mathbf{k}, t ; N_{c, \eta}\right)$ in Eq. (3), which enhances the HWM yield. The latter compensates for the reduction of the exciton binding energy due to the band filling such that no detuning from the exciton resonance occurs with the increase of the exciton density. Therefore, even in the strong field regime there is a considerable population of $1 s$-state; see Fig. 1. However, due to the reduction of the light-matter coupling strength, the $\mathrm{HHG} / \mathrm{HWM}$ yield is reduced for the resonant valley $(\tau=1)$.
Although excitons are driven with the left-handed circularly polarized light, in a high-intensity regime both valleys are excited, since the nonresonant part of the interband transition dipole moment due to the trigonal warping is large in the nonlinear interaction regime, especially for even-order nonlinearities [68]. As a result, there is a dominant contribution of even-order $(\ell+n)$ harmonics from the $\tau=-1$ valley; the spectra are shown in Appendix B. These harmonics remain larger when accounting for the dynamic screening [Fig. 3(a)], as $N_{c, \pm 1,-1}(\mathbf{k}, t) \ll 1$, and the Pauli blocking is negligible for this valley.

\section{CONCLUSION}

We have shown that the many-body electron-electron Coulomb interaction significantly alters HWM and HHG processes in MTMD nanostructures and is indispensable for a quantitative as well as a qualitative explanation of the output spectra. The Coulomb effect for the electron-hole recombination step makes a significant contribution to the enhancement of the HWM yield, which alters the interband and intraband mechanisms of HWM and reshapes spectra. These confirm that TMDC nanostructures can serve as an effective medium for the HWM.

\section{ACKNOWLEDGMENTS}

We thank Christoph H. Keitel for useful discussions. H.K.A. thanks the Max Planck Institute for Nuclear Physics for hospitality. This work was supported by the RA Science Committee in the frame of Research Project No. 18BL-020.

\section{APPENDIX A: EVOLUTIONARY EQUATION FOR A SINGLE-PARTICLE DENSITY MATRIX}

In this Appendix, we consider the details of the derivation of Eqs. (2) and (3) in the main text, and we provide concrete expressions for the interband transition dipole moment and the Berry connection. To proceed, we need the single-particle Hamiltonian. We will obtain this from the $\mathbf{k} \cdot \mathbf{p}$ model Hamiltonian up to the second order in $k$ (relative to $\tau K$ points) [69]:

$$
\widehat{H}(s, \tau ; \mathbf{k})=\left[\begin{array}{ll}
\mathcal{A}_{s \tau}(k) & \digamma_{s \tau}(\mathbf{k}) \\
\digamma_{s \tau}^{*}(\mathbf{k}) & \mathcal{B}_{s \tau}(k)
\end{array}\right],
$$

where

$$
\begin{gathered}
\mathcal{A}_{s \tau}(k)=\frac{\Delta_{\mathrm{DFT}}}{2}+\left|\Delta_{\mathrm{cb}}\right|+\beta_{s \tau} k^{2}+s \tau \Delta_{\mathrm{cb}}, \\
\mathcal{B}_{s \tau}(k)=-\frac{\Delta_{\mathrm{DFT}}}{2}+\alpha_{s \tau} k^{2}+(s \tau-1) \Delta_{\mathrm{vb}}, \\
\digamma_{s \tau}(\mathbf{k})=\gamma\left(\tau k_{x}-i k_{y}\right)+\varkappa_{s}\left(\tau k_{x}+i k_{y}\right)^{2},
\end{gathered}
$$

and $k^{2}=k_{x}^{2}+k_{y}^{2}, \Delta_{\mathrm{DFT}}, \gamma, \alpha_{s \tau}, \beta_{s \tau}$, and $\varkappa_{s}$ are energy parameters. The terms $s \tau \Delta_{\mathrm{cb}}$ and $s \tau \Delta_{\mathrm{vb}}$ are due to the SOCinduced coupling of valley $(\tau= \pm 1)$ and $\operatorname{spin}(s=1$ for $\uparrow z$ and $s=-1$ for $\downarrow_{z}$ ). The parameters of the Hamiltonian (A1) for $\mathrm{MoS}_{2}$ are shown in Table I. 
TABLE I. Parameters of the effective Hamiltonian.

\begin{tabular}{lc}
\hline \hline & $\mathrm{MoS}_{2}$ \\
\hline$\gamma(\mathrm{eV} \AA)$ & 2.76 \\
$\Delta_{\mathrm{DFT}}(\mathrm{eV})$ & 1.67 \\
$\alpha_{1}\left(\mathrm{eV} \AA^{2}\right)$ & -2.5 \\
$\alpha_{-1}\left(\mathrm{eV} \AA^{2}\right)$ & -2.05 \\
$\beta_{1}\left(\mathrm{eV} \AA^{2}\right)$ & 4.3 \\
$\beta_{-1}\left(\mathrm{eV} \AA^{2}\right)$ & 4.09 \\
$\varkappa_{1}\left(\mathrm{eV} \AA^{2}\right)$ & -1.48 \\
$\varkappa_{-1}\left(\mathrm{eV} \AA^{2}\right)$ & -1.45 \\
$\Delta_{\mathrm{cb}}(\mathrm{meV})$ & -1.5 \\
$\Delta_{\mathrm{vb}}(\mathrm{meV})$ & 74 \\
\hline \hline
\end{tabular}

There are four solutions in the energy spectrum of the Hamiltonian (A1). The eigenstates of the Hamiltonian (A1) for valence $(v)$ and conduction $(c)$ bands are

$$
\begin{aligned}
& |v, s, \tau ; \mathbf{k}\rangle=\frac{1}{\sqrt{1+\frac{\left|\digamma_{s \tau}(\mathbf{k})\right|^{2}}{\Delta_{s \tau}^{2}(\mathbf{k})}}}\left[\begin{array}{c}
-\frac{\digamma_{s \tau}(\mathbf{k})}{\Delta_{s \tau}(\mathbf{k})} \\
1
\end{array}\right], \\
& |c, s, \tau ; \mathbf{k}\rangle=\frac{1}{\sqrt{1+\frac{\left|\digamma_{s \tau}(\mathbf{k})\right|^{2}}{\Delta_{s \tau}^{2}(\mathbf{k})}}}\left[\begin{array}{c}
1 \\
\frac{\digamma_{s \tau}^{*}(\mathbf{k})}{\Delta_{s \tau}(\mathbf{k})}
\end{array}\right],
\end{aligned}
$$

where

$$
\begin{aligned}
\Delta_{s \tau}(\mathbf{k})= & \frac{\mathcal{A}_{s \tau}(k)-\mathcal{B}_{s \tau}(k)}{2} \\
& +\sqrt{\left(\frac{\mathcal{A}_{s \tau}(k)-\mathcal{B}_{s \tau}(k)}{2}\right)^{2}+\left|\digamma_{s \tau}(\mathbf{k})\right|^{2}} .
\end{aligned}
$$

The corresponding energies are

$$
\begin{aligned}
\mathcal{E}_{v s \tau}(\mathbf{k})= & \frac{\mathcal{A}_{s \tau}(k)+\mathcal{B}_{s \tau}(k)}{2} \\
& -\sqrt{\left(\frac{\mathcal{A}_{s \tau}(k)-\mathcal{B}_{s \tau}(k)}{2}\right)^{2}+\left|\digamma_{s \tau}(\mathbf{k})\right|^{2}}, \\
\mathcal{E}_{c s \tau}(\mathbf{k})= & \frac{\mathcal{A}_{s \tau}(k)-\mathcal{B}_{s \tau}(k)}{2} \\
& +\sqrt{\left(\frac{\mathcal{A}_{s \tau}(k)-\mathcal{B}_{s \tau}(k)}{2}\right)^{2}+\left|\digamma_{s \tau}(\mathbf{k})\right|^{2}} .
\end{aligned}
$$

We will work in the second quantization formalism, expanding the fermionic field operators on the basis of states given in (A5) and (A6), that is,

$$
\widehat{\Psi}(\mathbf{r})=\sum_{\lambda, \mathbf{k}} \widehat{e}_{\lambda ; \mathbf{k}}|\lambda ; \mathbf{k}\rangle e^{i \mathbf{k} \cdot \mathbf{r}},
$$

where $\widehat{e}_{\lambda ; \mathbf{k}}\left(\widehat{e}_{\lambda ; \mathbf{k}}^{\dagger}\right)$ is the annihilation (creation) operator for an electron with momentum $\mathbf{k}$, and $\lambda=\{b, s, \tau\}$ is the set of quantum numbers (band, spin, and valley). The second quantized free-particle Hamiltonian will be

$$
\widehat{H}_{\text {free }}=\sum_{b, s, \tau, \mathbf{k}} \mathcal{E}_{b, \eta}(\mathbf{k}) \widehat{e}_{b, \eta ; \mathbf{k}}^{\dagger} \widehat{e}_{b, \eta ; \mathbf{k}},
$$

where $\mathcal{E}_{b, \eta}(\mathbf{k})(b=c, v ; \eta=\{s, \tau\})$ eigenenergies are given by the formulas (A8) and (A9).

The polarization operator in the light-matter interaction part can be represented as follows:

$$
\begin{aligned}
\widehat{\mathbf{P}}= & -i e \sum_{b, \eta, \mathbf{k}, \mathbf{k}^{\prime}} \delta_{\mathbf{k}^{\prime} \mathbf{k}} \partial_{\mathbf{k}^{\prime}} \widehat{e}_{b, \eta ; \mathbf{k}}^{+} \widehat{e}_{b, \eta ; \mathbf{k}^{\prime}} \\
& -e \sum_{b, s, \tau, \mathbf{k}} \mathbf{D}_{\mathrm{B}}(b, \eta ; \mathbf{k}) \widehat{e}_{b, \eta ; \mathbf{k}}^{+} \widehat{e}_{b, \eta ; \mathbf{k}} \\
& -\sum_{\eta, \mathbf{k}}\left(\mathbf{D}_{\mathrm{tr}}(\eta ; \mathbf{k}) \widehat{e}_{v, \eta ; \mathbf{k}}^{+} \widehat{e}_{c, \eta ; \mathbf{k}}+\text { H.c. }\right),
\end{aligned}
$$

where

$$
\begin{aligned}
\mathbf{D}_{\mathrm{tr}}(s, \tau ; \mathbf{k}) & =e\left\langle v, s, \tau ; \mathbf{k}\left|i \partial_{\mathbf{k}}\right| c, s, \tau ; \mathbf{k}\right\rangle \\
& \simeq \frac{i e}{\Delta_{s \tau}(0)}\left[\gamma(\tau \widehat{\mathbf{x}}+i \widehat{\mathbf{y}})+2 \tau \varkappa_{s} k e^{-i \tau \theta}(\tau \widehat{\mathbf{x}}-i \widehat{\mathbf{y}})\right]
\end{aligned}
$$

is the interband transition dipole moment and

$$
\begin{aligned}
\mathbf{D}_{\mathrm{B}}(b, s, \tau ; \mathbf{k}) & =\left\langle b, s, \tau ; \mathbf{k}\left|i \partial_{\mathbf{k}}\right| b, s, \tau ; \mathbf{k}\right\rangle \\
& =\varsigma_{b} \frac{\operatorname{Re}\left[\digamma_{s \tau}(\mathbf{k}) i \partial_{\mathbf{k}} \digamma_{s \tau}^{*}(\mathbf{k})\right]}{\Delta_{s \tau}^{2}(\mathbf{k})+\left|\digamma_{s \tau}(\mathbf{k})\right|^{2}}
\end{aligned}
$$

is the Berry connection $\left(\varsigma_{c}=1\right.$ and $\left.\varsigma_{v}=-1\right)$. With the help of the Berry connection, one can calculate also the Berry curvature,

$$
\mathbf{B}(s, \tau ; \mathbf{k})=\partial \mathbf{k} \times\left[\mathbf{D}_{\mathrm{B}}(c, s, \tau ; \mathbf{k})-\mathbf{D}_{\mathrm{B}}(v, s, \tau ; \mathbf{k})\right] .
$$

In the following, we are going to approximate the Berry connection with its value near the $\mathbf{k}=\mathbf{0}$ point. In the leading order of $k$, we have

$$
\mathbf{D}_{\mathrm{B}}(c, s, \tau ; \mathbf{k})-\mathbf{D}_{\mathrm{B}}(v, s, \tau ; \mathbf{k})=\tau \frac{B_{s \tau}}{2}\left(k_{y} \widehat{\mathbf{x}}-k_{x} \widehat{\mathbf{y}}\right),
$$

where

$$
B_{s \tau}=|\mathbf{B}(s, \tau ; \mathbf{0})|=\frac{4 \gamma^{2}}{\Delta_{s \tau}^{2}(0)}
$$

is the total Berry curvature of bands. Note that the basic functions (A5) and (A6) are gauge-dependent. One can perform a gauge transform $|b, s, \tau ; \mathbf{k}\rangle \rightarrow \exp (i \Phi(b, s, \tau ; \mathbf{k}))|b, s, \tau ; \mathbf{k}\rangle$, where $\Phi(b, s, \tau ; \mathbf{k})(b=c, v)$ are arbitrary phases. The latter will not change the eigenenergies, but will lead to different transition dipole moments and Berry connections. Hence, for gauge invariance of the final results with respect to arbitrary phase transformation of Bloch functions, one should keep Berry connection terms in the evolutionary equation for the single-particle density matrix.

The electrons interact through long-range Coulomb forces, and the Hamiltonian for electron-electron interactions can be written

$$
\begin{aligned}
\widehat{H}_{\mathrm{C}}= & \frac{1}{2} \sum_{\eta_{1} \eta_{2}} \sum_{\mathbf{q}, \mathbf{k}^{\prime}, \mathbf{k}} V_{2 \mathrm{D}}(\mathbf{q}) \digamma_{\eta_{1} \eta_{2}}\left(\mathbf{q}, \mathbf{k}^{\prime}, \mathbf{k}\right) \\
& \times \widehat{e}_{\eta_{1} ; \mathbf{k}^{\prime}+\mathbf{q}}^{\dagger} \widehat{\boldsymbol{e}}_{\eta_{2} ; \mathbf{k}-\mathbf{q}}^{\dagger} \widehat{\boldsymbol{e}}_{\eta_{2} ; \mathbf{k}} \widehat{e}_{\eta_{1} ; \mathbf{k}^{\prime}},
\end{aligned}
$$


where $V_{2 \mathrm{D}}(\mathbf{q})$ is the $2 \mathrm{D}$ interaction potential in the momentum space and

$$
\digamma_{\eta_{1} \eta_{2}}\left(\mathbf{q}, \mathbf{k}^{\prime}, \mathbf{k}\right)=\left\langle\eta_{1} ; \mathbf{k}^{\prime}+\mathbf{q} \| \eta_{1} ; \mathbf{k}^{\prime}\right\rangle\left\langle\eta_{2} ; \mathbf{k}-\mathbf{q} \| \eta_{2} ; \mathbf{k}\right\rangle .
$$

The Coulomb interaction part (A17) contains products of four fermionic operators. We will treat the Coulomb interaction within the scope of mean-field theory, reducing the Hamiltonian (A17) into the so-called mean-field Hamiltonian, which allows us to obtain the closed set of equations for the dynamic mean-field parameters: $\mathcal{P}_{s, \tau}(\mathbf{k}, t)=$ $\left\langle\widehat{e}_{c, s, \tau ; \mathbf{k}}^{+}(t) \widehat{e}_{v, s, \tau ; \mathbf{k}}(t)\right\rangle, \mathcal{N}_{c, s, \tau}(\mathbf{k}, t)=\left\langle\widehat{e}_{c, s, \tau ; \mathbf{k}}^{+}(t) \widehat{e}_{c, s, \tau ; \mathbf{k}}(t)\right\rangle$, and $\mathcal{N}_{v, s, \tau}(\mathbf{k}, t)=\left\langle\widehat{e}_{v, s, \tau ; \mathbf{k}}^{+}(t) \widehat{e}_{v, s, \tau ; \mathbf{k}}(t)\right\rangle$. The following decomposition of a product of four fermionic operators has been made:

$$
\begin{aligned}
\left.\widehat{a}_{\alpha}^{\dagger} \widehat{b}_{\beta}^{\dagger} \widehat{c}_{\gamma} \widehat{d}_{\delta}\right|_{\text {Fock }}= & -\widehat{a}_{\alpha}^{\dagger} \widehat{c}_{\gamma}\left\langle\widehat{b}_{\beta}^{\dagger} \widehat{d}_{\delta}\right\rangle-\widehat{b}_{\beta}^{\dagger} \widehat{d}_{\delta}\left\langle\widehat{a}_{\alpha}^{\dagger} \widehat{c}_{\gamma}\right\rangle \\
& +\left\langle\widehat{a}_{\alpha}^{\dagger} \widehat{c}_{\gamma}\right\rangle\left\langle\widehat{b}_{\beta}^{\dagger} \widehat{d}_{\delta}\right\rangle .
\end{aligned}
$$

Taking into account the mean-field parameters and Eq. (A19) for the Coulomb part, we have

$$
\begin{aligned}
H_{\mathrm{MFC}}= & -\sum_{\eta_{1} \eta_{2}} \sum_{\mathbf{k}^{\prime}, \mathbf{k}} V_{2 \mathrm{D}}\left(\mathbf{k}-\mathbf{k}^{\prime}\right) \digamma_{\eta_{1} \eta_{2}}\left(\mathbf{k}-\mathbf{k}^{\prime}, \mathbf{k}^{\prime}, \mathbf{k}\right) \\
& \times\left\langle\widehat{e}_{\eta_{2} ; \mathbf{k}^{\prime}}^{\dagger} \widehat{e}_{\eta_{1} ; \mathbf{k}^{\prime}}\right\rangle \widehat{e}_{\eta_{1} ; \mathbf{k}}^{\dagger} \widehat{e}_{\eta_{2} ; \mathbf{k}},
\end{aligned}
$$

where

$$
\digamma_{\eta_{1} \eta_{2}}\left(\mathbf{k}^{\prime}, \mathbf{k}\right)=\left\langle\eta_{1} ; \mathbf{k} \| \eta_{1} ; \mathbf{k}^{\prime}\right\rangle\left\langle\eta_{2} ; \mathbf{k}^{\prime} \| \eta_{2} ; \mathbf{k}\right\rangle \delta_{s_{1} s_{2}} \delta_{\tau_{1} \tau_{2}} .
$$

In (A20) we have omitted the $c$-number. Now, from the Heisenberg equation

$$
i \hbar \frac{\partial \widehat{e}_{\eta_{2}, \mathbf{k}}^{\dagger} \widehat{e}_{\eta_{1}, \mathbf{k}}}{\partial t}=\left[\widehat{e}_{\eta_{2}, \mathbf{k}}^{\dagger} \widehat{e}_{\eta_{1}, \mathbf{k}}, \widehat{H}\right],
$$

where the Coulomb part of the total Hamiltonian $\widehat{H}$ is taken in the mean-field approximation (A20), one can obtain the closed set of evolutionary equations for $N_{c, s, \tau}(\mathbf{k}, t)$ and $\mathcal{P}_{s, \tau}(\mathbf{k}, t)$ :

$$
\begin{aligned}
i \hbar \frac{\partial \mathcal{P}_{s, \tau}(\mathbf{k}, t)}{\partial t}-i e \mathbf{E}(t) \frac{\partial \mathcal{P}_{s, \tau}(\mathbf{k}, t)}{\partial \mathbf{k}} & \\
= & \left\{\mathcal{E}_{v, s, \tau}(\mathbf{k})-\mathcal{E}_{c, s, \tau}(\mathbf{k})-\Sigma_{s, \tau}(\mathbf{k})-i \hbar \Gamma_{s, \tau}+e \mathbf{E}(t)\left[\mathbf{D}_{\mathrm{B}}(v, s, \tau ; \mathbf{k})-\mathbf{D}_{\mathrm{B}}(c, s, \tau ; \mathbf{k})\right]\right\} \mathcal{P}_{s, \tau}(\mathbf{k}, t) \\
& +\left(\sum_{\mathbf{k}^{\prime}} V_{2 \mathrm{D}}\left(\mathbf{k}-\mathbf{k}^{\prime}\right)\left[\digamma_{c c ; s, \tau}\left(\mathbf{k}^{\prime}, \mathbf{k}\right)+\digamma_{v v ; s, \tau}\left(\mathbf{k}^{\prime}, \mathbf{k}\right)\right] N_{c, s, \tau}\left(\mathbf{k}^{\prime}, t\right)\right) \mathcal{P}_{s, \tau}(\mathbf{k}, t) \\
& +\left(e \mathbf{E}(t) \mathbf{D}_{\mathrm{tr}}(s, \tau, \mathbf{k})-\sum_{\mathbf{k}^{\prime}} V_{2 \mathrm{D}}\left(\mathbf{k}-\mathbf{k}^{\prime}\right) \digamma_{v c ; s, \tau}\left(\mathbf{k}^{\prime}, \mathbf{k}\right) \mathcal{P}_{s, \tau}\left(\mathbf{k}^{\prime}, t\right)\right)\left[2 N_{c, s, \tau}(\mathbf{k}, t)-1\right], \\
i \hbar \frac{\partial N_{c, s, \tau}(\mathbf{k}, t)}{\partial t}-i e \mathbf{E}(t) \frac{\partial N_{c, s, \tau}(\mathbf{k}, t)}{\partial \mathbf{k}}=e \mathbf{E}(t) \mathbf{D}_{\mathrm{tr}}^{*}(s, \tau, \mathbf{k}) \mathcal{P}_{s, \tau}(\mathbf{k}, t)-i \hbar \Gamma_{0 s, \tau} N_{c, s, \tau}(\mathbf{k}, t) & -\sum_{\mathbf{k}^{\prime}} V_{2 \mathrm{D}}\left(\mathbf{k}-\mathbf{k}^{\prime}\right) \digamma_{v c ; s, \tau}^{*}\left(\mathbf{k}^{\prime}, \mathbf{k}\right) \mathcal{P}_{s, \tau}^{*}\left(\mathbf{k}^{\prime}, t\right) \mathcal{P}_{s, \tau}(\mathbf{k}, t)-\mathrm{c.c} .
\end{aligned}
$$

Here

$$
\Sigma_{s, \tau}(\mathbf{k})=\sum_{\mathbf{k}^{\prime}} V_{2 \mathrm{D}}\left(\mathbf{k}-\mathbf{k}^{\prime}\right) \digamma_{v v ; s, \tau}\left(\mathbf{k}^{\prime}, \mathbf{k}\right)
$$

is the Coulomb-induced self-energy, and we have introduced phenomenological dephasing rates $\Gamma_{s, \tau}$ and $\Gamma_{0 s, \tau}$ to account for other correlation terms neglected on the Hartree-Fock level. Note that the band gap is known to be underestimated by DFT, and its evaluation requires the use of $G W$ methodology. The DFT provides correct reduced masses and binding energies of excitons. Thus, we will fix the optical band gap, which is accessible in experiment [70]. The self-energy will be absorbed into the definition of the optical gap and will not be written explicitly hereafter. Since we are interested in the transitions via bound states, we will solve Eqs. (A22) and (A23) in the vicinity of the $K$ points. Thus, taking into account Eqs. (A8) and (A9), we will write

$$
\mathcal{E}_{c, s, \tau}(\mathbf{k})-\mathcal{E}_{v, s, \tau}(\mathbf{k})+\Sigma_{s \tau}(\mathbf{k})=\widetilde{\Delta}_{s \tau}+\frac{\hbar^{2} \mathbf{k}^{2}}{2 \mu_{s \tau}},
$$

where

$$
\mu_{s \tau}=\frac{\hbar^{2}}{2} \frac{\Delta_{s \tau}(0)}{\Delta_{s \tau}(0)\left(\beta_{s \tau}-\alpha_{s \tau}\right)+2 \gamma^{2}}
$$

is the reduced mass and

$$
\widetilde{\Delta}_{s \tau}=\Delta_{\mathrm{opt}}-\mathcal{E}_{A}+\Delta_{\mathrm{vb}}(1-s \tau)+(s \tau-1) \Delta_{\mathrm{cb}}
$$

is the renormalized gap. Here $\Delta_{\text {opt }}$ is the optical gap and $\mathcal{E}_{A}$ is the binding energy of the $A$ exciton $(s \tau=1)$.

The HWM is determined by the current density $\mathbf{j}=$ $\langle d \hat{\mathbf{P}} / d t\rangle$, which can be written as

$$
\mathbf{j}=-e\left(\frac{1}{i \hbar}\left[\widehat{\mathbf{r}}_{i}, \widehat{H}\right]+\frac{d}{d t}\left\langle\widehat{\mathbf{r}}_{\mathrm{B}}\right\rangle+\frac{d}{d t}\left\langle\widehat{\mathbf{r}}_{e}\right\rangle\right),
$$

where

$$
\widehat{\mathbf{r}}_{i}=i \sum_{\lambda, \mathbf{k}, \mathbf{k}^{\prime}} \delta_{\mathbf{k}^{\prime} \mathbf{k}} \partial_{\mathbf{k}^{\prime}} \widehat{e}_{\lambda ; \mathbf{k}}^{+} \widehat{e}_{\lambda ; \mathbf{k}^{\prime}}
$$

is the intraband part of the position operator,

$$
\widehat{\mathbf{r}}_{\mathrm{B}}=\sum_{\lambda, \mathbf{k}} \mathbf{D}_{\mathrm{B}}(\lambda ; \mathbf{k}) \widehat{e}_{\lambda ; \mathbf{k}}^{+} \widehat{e}_{\lambda ; \mathbf{k}}
$$




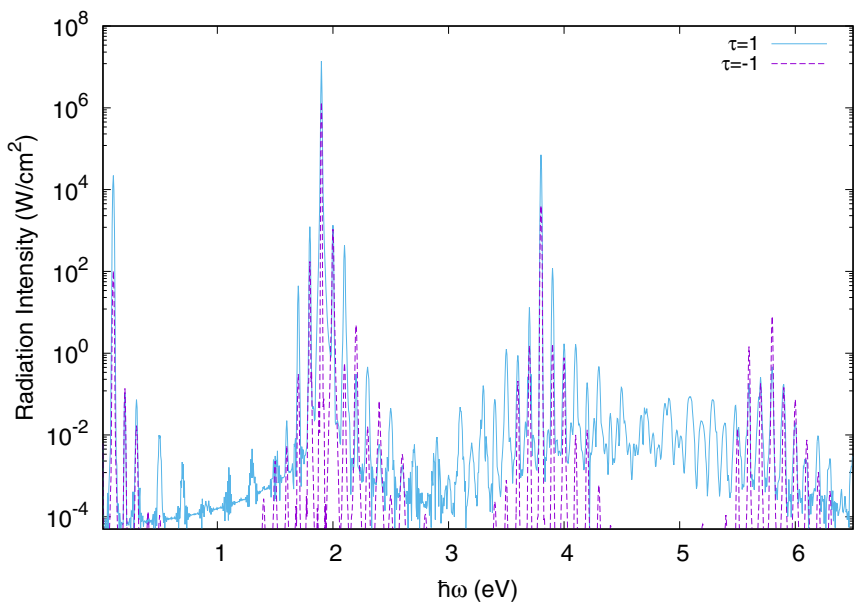

FIG. 4. Valley-resolved radiation spectra for the monolayer $\mathrm{MoS}_{2}$ on the $\mathrm{SiO}_{2}$ substrate with dynamic screening of the Coulomb interaction. The laser parameters are $\hbar \omega_{1}=1.9 \mathrm{eV}$ and $\hbar \omega_{2}=$ $0.1 \mathrm{eV}$, and $I_{1}=2.6 \times 10^{11} \mathrm{~W} / \mathrm{cm}^{2}$ and $I_{2}=2.2 \times 10^{9} \mathrm{~W} / \mathrm{cm}^{2}$.

is defined by the topology of bands, and the last term

$$
\widehat{\mathbf{r}}_{e}=\sum_{\eta, \mathbf{k}}\left(\mathbf{D}_{\mathrm{tr}}(\eta ; \mathbf{k}) \widehat{e}_{v, \eta ; \mathbf{k}}^{+} \widehat{e}_{c, \eta ; \mathbf{k}}+\text { H.c. }\right)
$$

defines interband current. Calculating $\left[\widehat{\mathbf{r}}_{i}, \widehat{H}\right]$, where the Coulomb part of the total Hamiltonian is treated in the Hartree-Fock approximation, one can obtain formulas for the interband $\mathbf{j}_{\mathrm{e}}(t)$ and intraband $\mathbf{j}_{\mathbf{a}}(t)$ currents used in the main text.

\section{APPENDIX B: VALLEY-RESOLVED RADIATION SPECTRA}

The first term in the interband transition dipole moment (A13) is responsible for selectively addressing valleys by left (right) -handed circularly polarized light. The second term in the square brackets in Eq. (A13) is due to the trigonal warping effect and is responsible for even-order nonlinear response. In particular, in the spectra of HWM near the fundamental frequency of the HW wave, the odd-order sidebands are due to the trigonal warping effect. Meanwhile, near the second harmonic of HW, the even-order harmonics are generated due to this term.

Although we derive excitons with the left-handed circularly polarized light, in a high-intensity regime both valleys are excited since the interband transition dipole moment $\mathbf{D}_{\text {tr }}(s, \tau ; \mathbf{k})$ consists of two parts-resonant $\sim(\tau \widehat{\mathbf{x}}+i \widehat{\mathbf{y}})$ and nonresonant $\sim(\tau \widehat{\mathbf{x}}-\imath \widehat{\mathbf{y}})-$ due to the trigonal warping effect (A13). The latter is small compared with the first one (see the parameters in Table I), and in the linear regime it is usually neglected. But for the nonlinear interaction regime, this term is crucial, especially for even-order nonlinear effects. The term in $\mathbf{D}_{\text {tr }}$ with the trigonal warping effect provides a resonance for the opposite valley $\tau=-1$, and as a result we have a dominant contribution of even-order $(\ell+n)$ harmonics from this valley (Fig. 4). In addition, since $N_{c, \pm 1,-1}(\mathbf{k}, t) \ll 1$, the Pauli blocking is negligible for this valley, and the evenorder harmonics remains larger also at the dynamic screening.
[1] P. Agostini and L. F. DiMauro, Rep. Prog. Phys. 67, 813 (2004).

[2] M. C. Kohler, T. Pfeifer, K. Z. Hatsagortsyan, and C. H. Keitel, Adv. At. Mol. Opt. Phys. 61, 159 (2012).

[3] P. B. Corkum and F. Krausz, Nat. Phys. 3, 381 (2007).

[4] F. Krausz and M. Ivanov, Rev. Mod. Phys. 81, 163 (2009).

[5] S. Ghimire, A. D. DiChiara, E. Sistrunk, P. Agostini, L. F. DiMauro, and D. A. Reis, Nat. Phys. 7, 138 (2011).

[6] B. Zaks, R. B. Liu, and M. S. Sherwin, Nature (London) 483, 580 (2012).

[7] F. Langer, M. Hohenleutner, C. P. Schmid, C. Poellmann, P. Nagler, T. Korn, C. Schüller, M. S. Sherwin, U. Huttner, J. T. Steiner, S. W. Koch, M. Kira, and R. Huber, Nature (London) 533, 225 (2016)

[8] O. Schubert, M. Hohenleutner, F. Langer, B. Urbanek, C. Lange, U. Huttner, D. Golde, T. Meier, M. Kira, S. W. Koch, and R. Huber, Nat. Photon. 8, 119 (2014).

[9] G. Vampa, C. R. McDonald, G. Orlando, D. D. Klug, P. B. Corkum, and T. Brabec, Phys. Rev. Lett. 113, 073901 (2014).

[10] G. Vampa, T. J. Hammond, N. Thire, B. E. Schmidt, F. Legare, C. R. McDonald, T. Brabec, and P. B. Corkum, Nature (London) 522, 462 (2015).

[11] G. Vampa, C. R. McDonald, G. Orlando, P. B. Corkum, and T. Brabec, Phys. Rev. B 91, 064302 (2015).

[12] G. Ndabashimiye, S. Ghimire, M. Wu, D. A. Browne, K. J. Schafer, M. B. Gaarde, and D. A. Reis, Nature (London) 534, 520 (2016).
[13] Y. S. You, D. A. Reis, and S. Ghimire, Nat. Phys. 13, 345 (2017).

[14] H. Liu, C. Guo, G. Vampa, J. L. Zhang, T. Sarmiento, M. Xiao, P. H. Bucksbaum, J. Vuckovic, S. Fan, and D. A. Reis, Nat. Phys. 14, 1006 (2018).

[15] S. A. Mikhailov and K. Ziegler, J. Phys. Condens. Matter 20, 384204 (2008).

[16] H. K. Avetissian, A. K. Avetissian, G. F. Mkrtchian, and Kh. V. Sedrakian, Phys. Rev. B 85, 115443 (2012).

[17] H. K. Avetissian, G. F. Mkrtchian, K. G. Batrakov, S. A. Maksimenko, and A. Hoffmann, Phys. Rev. B 88, 165411 (2013).

[18] P. Bowlan, E. Martinez-Moreno, K. Reimann, T. Elsaesser, and M. Woerner, Phys. Rev. B 89, 041408(R) (2014).

[19] I. Al-Naib, J. E. Sipe, and M. M. Dignam, Phys. Rev. B 90, 245423 (2014)

[20] L. A. Chizhova, F. Libisch, and J. Burgdorfer, Phys. Rev. B 94, 075412 (2016).

[21] H. K. Avetissian and G. F. Mkrtchian, Phys. Rev. B 94, 045419 (2016).

[22] L. A. Chizhova, F. Libisch, and J. Burgdorfer, Phys. Rev. B 95, 085436 (2017).

[23] D. Dimitrovski, L. B. Madsen, and T. G. Pedersen, Phys. Rev. B 95, 035405 (2017).

[24] N. Yoshikawa, T. Tamaya, and K. Tanaka, Science 356, 736 (2017). 
[25] H. K. Avetissian and G. F. Mkrtchian, Phys. Rev. B 97, 115454 (2018).

[26] H. K. Avetissian and G. F. Mkrtchian, Phys. Rev. B 99, 085432 (2019).

[27] A. K. Geim and I. V. Grigorieva, Nature (London) 499, 419 (2013).

[28] A. Splendiani, L. Sun, Y. Zhang, T. Li, J. Kim, C. Y. Chim, G. Galli, and F. Wang, Nano Lett. 10, 1271 (2010).

[29] K. F. Mak, C. Lee, J. Hone, J. Shan, and T. F. Heinz, Phys. Rev. Lett. 105, 136805 (2010).

[30] Y. Li, A. Chernikov, X. Zhang, A. Rigosi, H. M. Hill, A. M. van der Zande, D. A. Chenet, E.-M. Shih, J. Hone, and T. F. Heinz, Phys. Rev. B 90, 205422 (2014).

[31] D. Y. Qiu, F. H. da Jornada, and S. G. Louie, Phys. Rev. Lett. 111, 216805 (2013).

[32] C. Zhang, H. Wang, W. Chan, C. Manolatou, and F. Rana, Phys. Rev. B 89, 205436 (2014).

[33] G. Moody, C. K. Dass, K. Hao, C.-H. Chen, L.-J. Li, A. Singh, K. Tran, G. Clark, X. Xu, G. Berghäser, E. Malic, A. Knorr, and X. Li, Nat. Commun. 6, 8315 (2015).

[34] D. Xiao, M. C. Chang, and Q. Niu, Rev. Mod. Phys. 82, 1959 (2010).

[35] T. C. Berkelbach, M. S. Hybertsen, and D. R. Reichman, Phys. Rev. B 88, 045318 (2013).

[36] A. Ramasubramaniam, Phys. Rev. B 86, 115409 (2012).

[37] K. He, N. Kumar, L. Zhao, Z. Wang, K. F. Mak, H. Zhao, and J. Shan, Phys. Rev. Lett. 113, 026803 (2014).

[38] G. Berghäuser and E. Malic, Phys. Rev. B 89, 125309 (2014).

[39] M. M. Ugeda, A. J. Bradley, S. F. Shi, H. Felipe, Y. Zhang, D. Y. Qiu, W. Ruan, S. K. Mo, Z. Hussain, Z. X. Shen, and F. Wang, Nat. Mater. 13, 1091 (2014).

[40] A. Steinhoff, J-H. Kim, F. Jahnke, M. Rösner, D-S. Kim, C. Lee, G. H. Han, M. S. Jeong, T. O. Wehling, and C. Gies, Nano Lett. 15, 6841 (2015).

[41] G. Wang, A. Chernikov, M. M. Glazov, T. F. Heinz, X. Marie, T. Amand, and B. Urbaszek, Rev. Mod. Phys. 90, 021001 (2018).

[42] N. Kumar, S. Najmaei, Q. Cui, F. Ceballos, P. M. Ajayan, J. Lou, and H. Zhao, Phys. Rev. B 87, 161403(R) (2013).

[43] L. M. Malard, T. V. Alencar, A. P. M. Barboza, K. F. Mak, and A. M. de Paula, Phys. Rev. B 87, 201401(R) (2013).

[44] Y. Li, Y. Rao, K. F. Mak, Y. You, S. Wang, C. R. Dean, and T. F. Heinz, Nano Lett. 13, 3329 (2013).

[45] W. T. Hsu, Z. A. Zhao, L. J. Li, C. H. Chen, M. H. Chiu, P. S. Chang, Y. C. Chou, and W. H. Chang, ACS Nano 8, 2951 (2014).

[46] D. J. Clark, V. Senthilkumar, C. T. Le, D. L. Weerawarne, B. Shim, J. I. Jang, J. H. Shim, J. Cho, Y. Sim, M.-J. Seong, S. H. Rhim, A. J. Freeman, K.-H. Chung, and Y. S. Kim, Phys. Rev. B 90, 121409(R) (2014).

[47] C. Janisch, Y. Wang, D. Ma, N. Mehta, A. L. Elias, N. PereaLopez, M. Terrones, V. Crespi, and Z. Liu, Sci. Rep. 4, 5530 (2014).
[48] K. L. Seyler, J. R. Schaibley, P. Gong, P. Rivera, A. M. Jones, S. Wu, J. Yan, D. G. Mandrus, W. Yao, and X. Xu, Nat. Nanotech. 10, 407 (2015).

[49] G. T. Forcherio, J. Riporto, J. R. Dunklin, Y. Mugnier, R. Le Dantec, L. Bonacina, and D. K. Roper, Opt. Lett. 42, 5018 (2017).

[50] A. Autere et al., Phys. Rev. B 98, 115426 (2018).

[51] R. E. F. Silva, I. V. Blinov, A. N. Rubtsov, O. Smirnova, and M. Ivanov, Nat. Photon. 12, 266 (2018).

[52] T. T. Luu, M. Garg, S. Yu. Kruchinin, A. Moulet, M. Th. Hassan, and E. Goulielmakis, Nature (London) 521, 498 (2015).

[53] J.-Y. Yan, Phys. Rev. B 78, 075204 (2008).

[54] J. A. Crosse and R. B. Liu, Phys. Rev. B 89, 121202(R) (2014).

[55] X. T. Xie, B. F. Zhu, and R. B. Liu, New J. Phys. 15, 105015 (2018).

[56] H. Liu, Y. Li, Y. S. You, S. Ghimire, T. F. Heinz, and D. A. Reis, Nat. Phys. 13, 262 (2017).

[57] N. Yoshikawa, K. Nagai, K. Uchida, Y. Takaguchi, S. Sasaki, Y. Miyata, and K. Tanaka, Nat. Commun. 10, 1 (2019).

[58] F. Langer, C. P. Schmid, S. Schlauderer, M. Gmitra, J. Fabian, P. Nagler, C. Schüller, T. Korn, P. G. Hawkins, J. T. Steiner, U. Huttner, S. W. Koch, M. Kira, and R. Huber, Nature (London) 557, 76 (2018).

[59] A. Jimenez-Galan, R. E. F. Silva, O. Smirnova, and M. Ivanov, arXiv:1910.07398.

[60] M. Kira and S. W. Koch, Semiconductor Quantum Optics (Cambridge University Press, Cambridge, 2012).

[61] L. V. Keldysh, Pis'ma Zh. Eksp. Teor. Fiz. 29, 716 (1979) [JETP Lett. 29, 658 (1979)].

[62] P. Cudazzo, I. V. Tokatly, and A. Rubio, Phys. Rev. B 84, 085406 (2011).

[63] L. V. Keldysh, J. Exp. Theor. Phys. 47, 1945 (1964) [Sov. Phys. JETP 20, 1307 (1965)].

[64] E. Malic, T. Winzer, E. Bobkin, and A. Knorr, Phys. Rev. B 84, 205406 (2011).

[65] A. Steinhoff, M. Florian, M. Rösner, M. Lorke, T. O. Wehling, C. Gies, and F. Jahnke, 2D Mater. 3, 031006 (2016).

[66] H. Haug and S. W. Koch, Quantum Theory of the Optical and Electronic Properties of Semiconductors (World Scientific, Singapore, 2009).

[67] P. B. Corkum, Phys. Rev. Lett. 71, 1994 (1993).

[68] G. F. Mkrtchian, A. Knorr, and M. Selig, Phys. Rev. B 100, 125401 (2019).

[69] A. Kormanyos, G. Burkard, M. Gmitra, J. Fabian, V. Zolyomi, N. D. Drummond, and V. Fal'ko, 2D Mater 2, 049501 (2015).

[70] D. Christiansen, M. Selig, G. Berghauser, R. Schmidt, I. Niehues, R. Schneider, A. Arora, S. M. de Vasconcellos, R. Bratschitsch, E. Malic, and A. Knorr, Phys. Rev. Lett. 119, 187402 (2017). 